\title{
A national survey on use of less invasive surfactant administration in Turkey
}

\author{
Mehmet Yekta Öncel ${ }^{1,2}$, Ömer Erdeve $^{3 \oplus}$ \\ ${ }^{1}$ Department of Pediatrics, Division of Neonatology, İzmir Katip Çelebi University Faculty of Medicine; ${ }^{2}$ Division of Neonatology, \\ Tepecik Training and Research Hospital, Izmir; ${ }^{3}$ Department of Pediatrics, Division of Neonatology, Ankara University Faculty of \\ Medicine, Ankara, Turkey.
}

\begin{abstract}
Background. The aim of the study was to assess the rate of utilization, policy of premedication, technique, equipment, experience on safety and efficacy for less invasive surfactant administration or minimally invasive surfactant therapy (LISA/MIST) use in Turkey.

Methods. An online survey was designed and distributed via Google Forms tool to 350 neonatologists from 173 units through NICU-Turk mailing list of the Turkish Neonatal Society. Participants were asked to answer the survey for their own neonatal intensive care unit (NICU).
\end{abstract}

Results. LISA/MIST use rate was $81.6 \%$ among 87 NICUs which responded (response rate was $50.2 \%$ ). LISA was used regularly in 23 of the units $(26.4 \%)$, occasionally in $35(40.2 \%)$, rarely in $12(13.8 \%)$, and only for clinical trials in 1 (1.1\%). LISA/MIST has been never applied in 16 units (18.4\%).

Conclusions. LISA/MIST is widely used in Turkey similar to several regions in Europe but unlike the USA. Future studies are expected to further clarify some questions about LISA/MIST procedure, especially on its efficacy and safety.

Key words: less invasive surfactant administration, minimally invasive surfactant therapy, respiratory distress syndrome, surfactant, survey.

Preterm infants requiring surfactant replacement are typically treated by using the INSURE (INtubation SURfactant Extubation) technique. Recently, less invasive surfactant administration or minimally invasive surfactant therapy (LISA/MIST) has come into use in neonatal practice. ${ }^{1,2}$ Encouraging results from the initial small feasibility studies were followed by larger randomized controlled trials (RCTs). The first RCT including 12 German neonatal intensive care units (NICU) found that surfactant administration via a thin catheter reduced the need for intubation and mechanical ventilation $(\mathrm{MV}) .{ }^{1}$ Later on, our group compared the LISA technique to INSURE and reported

\footnotetext{
$\bowtie$ Mehmet Yekta Öncel

dryekta@gmail.com
}

Received 18th July 2019, revised 1st September 2019, accepted 13th September 2019. lower rates of both MV and bronchopulmonary dysplasia (BPD) in preterm infants who received surfactant by LISA method. ${ }^{2}$ A systematic review of 30 trials (5598 neonates) examining ventilation strategies demonstrated that use of LISA was associated with lower odds of the composite outcome of death or BPD and severe intraventricular hemorrhage (IVH) in preterm infants. Calculation of the ranking possibilities found LISA to be the best strategy in this group of infants. ${ }^{3}$ Similarly, another systematic review of six RCTs demonstrated that LISA use in infants with respiratory distress syndrome (RDS) was associated with reduced incidences for need for MV and BPD/death at 36 weeks of gestation. ${ }^{4}$ LISA/MIST has been recommended for the administration of surfactant in addition to INSURE technique, with an advantage not needing intubation. LISA has started to come to the forefront with low BPD and/or mortality rates in recent years. ${ }^{3,4}$ 
A survey of LISA use across Europe showed variations in equipment used and techniques applied for LISA as well as different views on the indications and perceived efficacy of this intervention. ${ }^{5}$ Only five NICUs from Turkey participated in this survey, therefore we did not have any idea on the current practice regarding LISA/MIST in Turkey.

The aim of our survey trial was to assess the rate of utilization, policy of premedication, perceptions of indication and feasibility in regard to gestational age, technique, and equipment as well as experience of safety and efficacy used for LISA/MIST.

\section{Material and Methods}

An online survey was designed by using the GoogleFormstool.WecontactedallNICUswithin the Turkish Neonatal Society database (NICUTurk) and requested that the questionnaire be completed by a single representative from each NICU. Representatives were neonatologist who regularly worked as attendings in the NICU. The study was approved by the local ethics committee (2018/16-4). Informed consent was obtained from the participants.

The first ten questions focused on general information regarding participants' demographic data, knowledge and used LISA/ MIST in their NICUs. Subsequently, participants who used LISA/MIST were routed to additional usage-specific questions (seventeen) about LISA/MIST. Similarly, the questionnaire was completed by asking 3 questions for those who did not use LISA/MIST.

\section{Statistical Analyses}

Data analysis was descriptive. Categorical variables are presented in absolute numbers and percentages, and quantitative data are reported as median and minimum-maximum. Data analysis was performed using SPSS Version 21 (IBM Corp, Armonk, New York).

\section{Results}

Our survey was sent to all neonatologists $(n=350)$ who are included in the NICU-Turk mailing list of Turkish Neonatal Society. One responsible person from each unit was asked to complete the questionnaire. Ninety-seven neonatologists answered the survey, but since two people from 10 centers were from the same center, the answers of the senior neonatologists were accepted and included in the analysis. Of the 173 NICUs 87 (50.2\%) answered the survey, which represented 195 out of 350 neonatologists (55.7\%).

Participation rate of academic and public (University Hospitals, Training and Research Hospitals) institutions (61/82, 70.1\%) was quite high whereas participation from private hospitals was low $(11 / 91,12.1 \%)$. The median number of incubators in these centers was 27 (6-120) and median number of neonatologists was 2 (1-12). The median number of newborns under $1500 \mathrm{~g}$ infants per year in participating NICUs was 82 (2-1000).

Seventy percent of the responding neonatologists were working in an academic (University Hospital and Training and Research Hospital) setting. The huge majority (90.8\%) was from level III-IV NICUs, and 77\% of the respondents were administering surfactant at least 50 times per year (Table I). According to the survey results, LISA/MIST was used by 71 of the responding neonatologists (81.6\%). Figure 1 shows that the frequency of LISA/MIST use.

Among those who were using LISA/MIST, 52.1\% had a protocol for its use. The vast majority (95.8\%) did not use sedation/premedication with LISA/MIST. The majority (59 NICUs, $83.1 \%)$ used the feeding tube, six $(8.45 \%)$ an angiocath, five $(7 \%)$ a vascular catheter and one $(8.6 \%)$ suction catheter for the application. Almost all neonatologists (97.2\%) inserted the catheter orally and only $12.7 \%$ used Magill forceps. Seventy four percent of respondents gave $200 \mathrm{mg} / \mathrm{kg}$ of surfactant and the majority $(54.9 \%)$ gave it over $30 \mathrm{sec}-1 \mathrm{~min}$, followed 
Table I. Basic questions and answers within the survey.

Questions and answers Number of respondents $(n=87)$

Type of hospital

University Hospital

$38(43.7)$

Training and Research Hospital

$23(26.4)$

Public Hospital

$15(17.2)$

Private Hospital

$11(12.6)$

Level of your center

Level II

Level III

$63(72.4)$

Level IV

$16(18.4)$

How many times per year is surfactant administered in your center?

$<20$

20-50

50-100

$32(36.8)$

$>100$

$35(40.2)$

Do you use any non-invasive surfactant administration (LISA/MIST) techniques in your center?

Yes

No $16(18.4)$

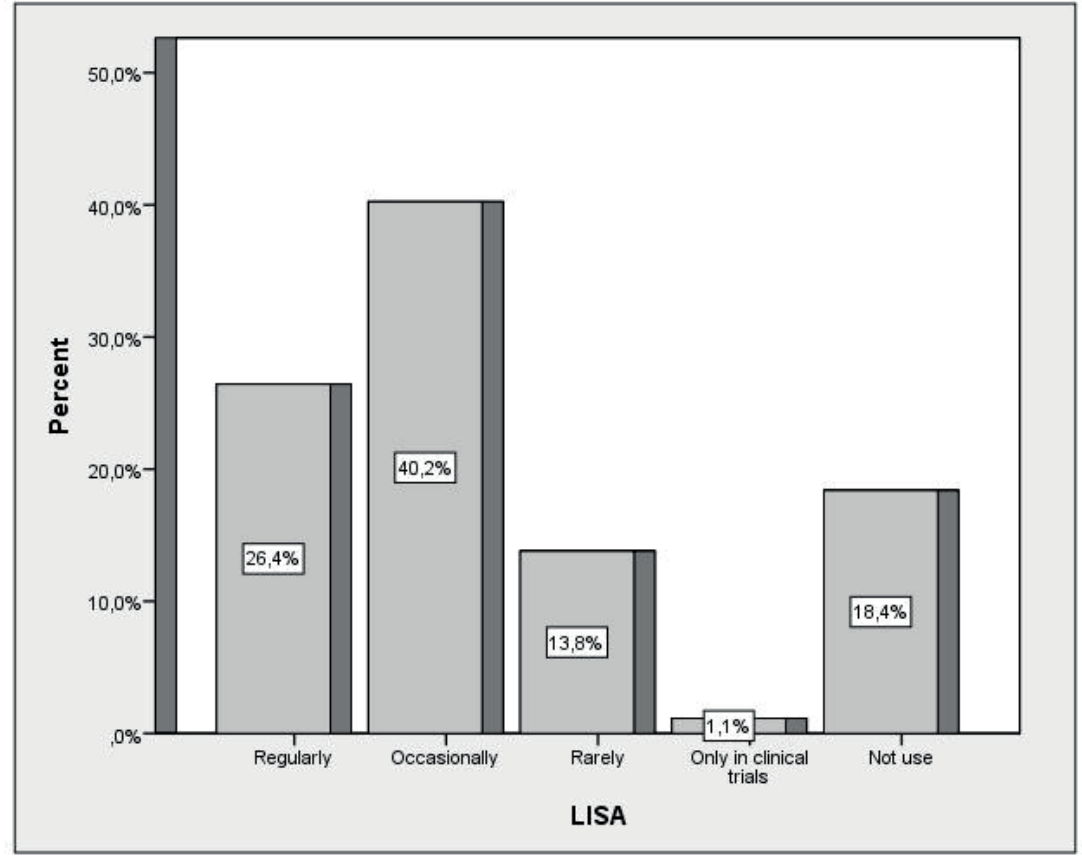

Fig. 1. LISA/MIST use frequencies in Turkey. 
by $21.1 \%$ in $<30 \mathrm{sec}$. The most preferred noninvasive ventilation mode during LISA/ MIST was nasal intermittent positive pressure ventilation (NIPPV) (57.7\%) (Table II).

Adverse events of LISA/MIST were experienced by $84.5 \%$ of neonatologists. Tracheal surfactant reflux $(76.1 \%)$, bradycardia $(26.8 \%)$, hypoxia (22.5\%) and need for intubation (19.7\%) among others were observed. Perceived efficacy and safety of LISA/MIST among participating neonatologists were high as $52.1 \%$ and $81.7 \%$, respectively. Twenty nine percent of the respondents believed that the current literature on LISA/MIST is strong enough to recommend it as standard care, $14 \%$ of respondents felt that the literature is not strong enough, $47.9 \%$ believed more evidence is needed and the remaining $8.5 \%$ were not sure (Table III).

LISA/MIST has never been applied in 16 NICUs (18.4\%). Leading reasons for not using LISA/MIST were lack of experience (50\%) and inconclusive evidence for the use of LISA/MIST ( $25 \%)$. INSURE $(75 \%)$ or intubation $(25 \%)$ was used for surfactant therapy instead in these NICUs. Majority of neonatologists not applying LISA/MIST (87.5\%) reported that they would consider utilizing it in the future (Table IV).

\section{Discussion}

Recently, LISA has been promoted as an alternative method for surfactant administration. Several reports have shown that LISA is becoming established in daily practice. $^{1,4}$ Many guidelines have accepted LISA as standard care in a selected group of premature infants. The recent European Consensus Guidelines and Turkish Guidelines on surfactant use recommended LISA as the preferred mode of surfactant administration for spontaneously breathing babies on continuous positive airway pressure (CPAP), providing that clinicians are experienced with this technique (level of evidence: B2). ${ }^{6,7}$ However, there is still a wide variation regarding many aspects of the LISA/MIST method. ${ }^{4}$ We aimed to investigate the neonatologists' knowledge, attitude and practices regarding the LISA/MIST approach. In addition to general information on this topic, our survey was carefully structured to examine LISA/MIST usage specific questions for those who do not use LISA/MIST.

Seventy percent of the respondents came from academic and public institutions with the vast majority practicing in level III and IV NICUs that commonly use surfactant in their practice. The utilization rate of LISA $(81.6 \%)$ in this survey was one of the highest reported to date in the literature, just after the rate reported from Spain (89\%). ${ }^{8}$ Indeed, the rate of regular use of LISA in our survey was only $26.4 \%$ but the high rate was due to additional use in NICUs beside INSURE technique. In a recent large scale survey of 37 European countries, the mean utilization rate was $52 \%,{ }^{5}$ and previous studies reported even lower use. A national statistical analysis in Poland in 2015 reported that LISA was used in only $1 \%$ of hospitals. ${ }^{9}$ Nordic hospitals had a utilization rate of $32 \%$ in a survey conducted in 2016. ${ }^{10}$ Recent surveys from European countries such as Spain $^{8}$ and Turkey (our study) show that LISA use rates have increased since 2015. Thus, we believe that within a few years the technique will be widely used in other NICUs. On the contrary, LISA usage in the USA and UK have been found to be $15 \%$ and $18.7 \%$ in new surveys. ${ }^{11,12}$ The authors thought that the reason for the low rate of LISA use in these countries was due to the lack of structured training in the LISA method.

LISA/MIST usage specific questions revealed that fifty percent of the respondents have written a protocol for LISA use. Intrestingly, the majority of neonatologists consider LISA even in extremely immature infants. This may be in line with results of a recent study in infants at 23-26 weeks gestational age, where increased survival without major complications was seen in the group of infants treated with LISA. However, subgroup analysis from this study revealed the most benefit in the more mature infants of 25 and 26 weeks. ${ }^{13}$ According to the results of our survey, although $33.8 \%$ of NICUs 
Table II. LISA/MIST usage-specific questions and answers within the survey.

Questions and answers

If you are using LISA/MIST, do you have a protocol for its use?

Yes

No

What is the preferred catheter in LISA/MIST?

Feeding tube

Vascular catheter

Angiocath (Hobart method)

Suction catheter

Do you shorten the catheter in LISA/MIST?

Yes

No

Which intubation method do you use in LISA/MIST?

Oral

Nasal

Do you use Magill forceps in LISA/MIST?

Yes

No

Do you use any sedation/premedication when using LISA/MIST?

Yes

Benzodiazepines

Atropine

Opioids

No

How much time do you allow for surfactant delivery when using LISA/MIST?

$<30 \mathrm{sec}$

$30 \mathrm{sec}-1 \mathrm{~min}$

$1-2 \mathrm{~min}$

$2-3 \mathrm{~min}$

$>3$ min

Which is the most preferred gestational age to use LISA/MIST?

All gestational age

$<24 \mathrm{w}$

$24-26 \mathrm{w}$

$26-28 \mathrm{w}$

$28-32 w$

$>32 \mathrm{w}$

Which is the most preferred time interval to use LISA/MIST?

Every time

$0-6 \mathrm{~h}$

$6-12 \mathrm{~h}$

$12-24 h$

$>24 \mathrm{~h}$
Number of respondents $(\mathrm{n}=71)$

$34(47.9)$

$59(83.1)$

5 (7)

$6(8.45)$

1 (1.4)

43 (60.6)

28 (39.4)

69 (97.2)

$2(2.8)$

9 (12.7)

62 (87.3)

$3(4.2)$

3

-

68 (95.8)

15 (21.1)

39 (54.9)

7 (9.9)

10 (14.1)

24 (33.8)

$6(8.5)$

15 (21.1)

21 (29.6)

5 (7)

24 (33.8)

2 (2.8)

1 (1.4)

LISA/MIST: less invasive surfactant administration or minimally invasive surfactant therapy, CPAP: continuous positive airway pressure; NIPPV: nasal intermittent positive pressure ventilation; BIPAP: bilevel positive airway pressure 
Table II. Continued.

Questions and answers Number of respondents $(\mathrm{n}=71)$

Which surfactant do you prefer to use for LISA/MIST?

Poractant alfa

$67(94.4)$

Beractant

Calfactant

Which dose of surfactant do you prefer to use for LISA/MIST?

$100 \mathrm{mg} / \mathrm{kg}$

$11(15.5)$

$200 \mathrm{mg} / \mathrm{kg}$

$53(74.6)$

Other

$7(9.8)$

Would you consider LISA/MIST again if an infant needs a repeat dose of surfactant after 6-12 h?

Yes

No

Which non-invasive ventilation method do you prefer during LISA/MIST?

Ventilator-derived nasal CPAP

Bubble CPAP

NIPPV

$41(57.7)$

BIPAP

$1(1.4)$

LISA/MIST: less invasive surfactant administration or minimally invasive surfactant therapy, CPAP: continuous positive airway pressure; NIPPV: nasal intermittent positive pressure ventilation; BIPAP: bilevel positive airway pressure

Table III. Adverse events, perceived efficacy and safety during LISA/MIST.

\begin{tabular}{lc}
\hline Parameters & $\mathrm{n}(\%)$ \\
\hline Adverse events & $11(15.5)$ \\
None & $54(76.1)$ \\
Tracheal surfactant reflux & $19(26.8)$ \\
Bradycardia & $16(22.5)$ \\
Hypoxia & $14(19.7)$ \\
Need for intubation & $8(11.3)$ \\
Unilateral surfactant administration & $2(2.8)$ \\
Other (gastric deposition, airway obstruction) & \\
Perceived efficacy & $8(11.3)$ \\
Very high & $37(52.1)$ \\
High & $24(33.8)$ \\
Medium & $2(2.8)$ \\
Low & - \\
Very low & \\
Perceived safety & $58(81.7)$ \\
Yes & $1(1.4)$ \\
No & $12(16.9)$ \\
Not sure & \\
Do you believe that the current literature on LISA/MIST is strong enough to & \\
recommend it as standard care? & \\
Yes & $21(29.6)$ \\
No & $10(14.1)$ \\
More evidence is needed & $34(47.9)$ \\
Not sure & $6(8.5)$ \\
\hline
\end{tabular}


Table IV. LISA/MIST non usage-specific questions and answers within the survey.

\begin{tabular}{lc}
\hline Questions and answers & Number of respondents $(\mathrm{n}=16)$ \\
\hline If you do not use the LISA/MIST method, which method do you prefer more? & \\
Intubated & $4(25)$ \\
INSURE & $12(75)$ \\
Why do you not use the LISA/MIST method? & $8(50)$ \\
Lack of experience & $4(25)$ \\
Inconclusive evidence for the use of LISA & $4(25)$ \\
Other (missing consensus, low efficacy, adverse events, congestion) & \\
Do you consider utilizing the LISA/MIST method in the future? & $14(87.5)$ \\
Yes & $2(12.5)$ \\
No & \\
\hline
\end{tabular}

LISA/MIST: less invasive surfactant administration or minimally invasive surfactant therapy, INSURE: INtubation SURfactant Extubation

preferred LISA/MIST irrespective of gestational age, generally mentioned preferred weeks were 28-32 (29.6\%) and 26-28 (21.1\%). The LISA/MIST usage rate for $24-26$ weeks was only $8.5 \%$.

Sedation or premedication use rates vary widely among NICUs. For example, $52 \%$ of neonatologists did not use any premedication for performing LISA in the European survey. ${ }^{5}$ Unexpectedly, very few babies (4.2\%) received sedation before the procedure in our survey results. Refrainment from sedative use may be due to the possible complications. We think that compared to invasive intubation some neonatologists perceive LISA/MIST as being less traumatic or maintenance of spontaneous breathing being superior to analgesia or sedation. Most physicians seem to use a feeding tube $(83.1 \%)$ in our survey. Compared to the European survey, the rate of use of a feeding tube seems to be quite high in our study. In both European and USA surveys, angiocath (34\% and $20.3 \%$ ) was the second most frequently used method after the feeding tube $(56 \%$ and 46.3\%). ${ }^{5,11}$ Magill forceps for placement use rate was $65 \%$ in the European survey ${ }^{5}$ whereas only a minority of neonatologists (12.7\%) used Magill forceps to facilitate endotracheal placement of the catheter in our survey. Overall in the literature, the majority of neonatologists distribute the surfactant fairly slowly, over $1 \mathrm{~min}$ or more. ${ }^{5,11}$ On the contrary, the more preferred time in our survey was 30 sec1 min, which may explain higher tracheal surfactant reflux rate. Other major adverse events included bradycardia and hypoxia. The most preferred non-invasive method seems to be NIPPV as unexpected when we compare with the nasal CPAP rate in other surveys. NIPPV has been shown to reduce the need for intubation recently, which may be a reason for its preference in our survey. ${ }^{14}$

There are some limitations in our survey. First, the response rate was $50.2 \%$. Although, participation rate of academic and public institutions was quite high. Most responses came from level III-IV NICUs with high patient loads. Second, we contacted one person from each unit, so the answers might have reflected unit policy rather than personal views. Third, majority of the responders said that they used LISA/MIST occasionally. Although the frequency of LISA/MIST use in the survey could be answered as "regularly, occasionally, rarely, only in clinical trials and not use", "regularly, occasionally and rarely" words might have different meanings for each respondent or unit because each NICU had a different number of incubator and different criteria for LISA/MIST use.

In conclusion, LISA/MIST has become an increasingly common practice in recent years. This technique is widely applied in European 
NICUs including Turkey, unlike the USA. Future studies are expected to further clarify some questions regarding patient selection, the type of thin catheter use, time of administration, non-invasive ventilation method during LISA, sedation/premedication, procedural efficacy and safety of LISA. We think that each country should evaluate its own data according to their settings.

\section{Acknowledgements}

Special thanks to members of Turkish Neonatal Society who filled out the questionnaire.

\section{REFERENCES}

1. Göpel W, Kribs A, Ziegler A, et al; German Neonatal Network Collaborators. Avoidance of mechanical ventilation by surfactant treatment of spontaneously breathing preterm infants (AMV): an open-label, randomised, controlled trial. Lancet 2011; 378: 16271634.

2. Kanmaz HG, Erdeve O, Canpolat FE, Mutlu B, Dilmen U. Surfactant administration via thin catheter during spontaneous breathing: randomized controlled trial. Pediatrics 2013; 131: e502-e509.

3. Isayama $\mathrm{T}$, Iwami $\mathrm{H}, \mathrm{McD}$ onald $\mathrm{S}$, Beyene $\mathrm{J}$. Association of noninvasive ventilation strategies with mortality and bronchopulmonary dysplasia among preterm infants: a systematic review and meta-analysis. JAMA 2016; 316: 611-624.

4. Aldana-Aguirre JC, Pinto M, Featherstone RM, Kumar M. Less invasive surfactant administration versus intubation for surfactant delivery in preterm infants with respiratory distress syndrome: a systematic review and meta-analysis. Arch Dis Child Fetal Neonatal Ed 2017; 102: F17-F23.

5. Klotz D, Porcaro U, Fleck T, Fuchs H. European perspective on less invasive surfactant administration-a survey. Eur J Pediatr 2017; 176: 147-154.
6. Sweet DG, Carnielli V, Greisen G, et al. European Consensus Guidelines on the management of respiratory distress syndrome-2019 update. Neonatology 2019; 115: 432-450.

7. Özkan H, Erdeve Ö, Kanmaz Kutman HG. Turkish Neonatal Society guideline on the management of respiratory distress syndrome and surfactant treatment. Turk Pediatri Ars 2018; 53(Suppl 1): S45-S54.

8. Fernandez C, Boix H, Camba F, Comuñas JJ, Castillo F. Less invasive surfactant administration in Spain: a survey regarding its practice, the target population, and premedication use. Am J Perinatol 2020; 37: 277 280 .

9. Borszewska-Kornacka MK, Kostuch M, Korbal P, Krajewski P; Polish RDS Study Group. Strategies of using surfactant results of the first polish national survey of daily practice. Dev Period Med 2015; 19(3 Pt 1): 271-276.

10. Heiring C, Jonsson B, Andersson S, Björklund LJ. Survey shows large differences between the Nordic countries in the use of less invasive surfactant administration. Acta Paediatr 2017; 106: 382-386.

11. Kurepa D, Perveen S, Lipener Y, Kakkilaya V. The use of less invasive surfactant administration (LISA) in the United States with review of the literature. J Perinatol 2019; 39: 426-432.

12. Jeffreys E, Hunt K, Dassios T, Greenough A. UK survey of less invasive surfactant administration. Arch Dis Child Fetal Neonatal Ed 2019; 104: F567.

13. Kribs A, Roll C, Göpel W, et al; NINSAPP Trial Investigators Nonintubated surfactant application vs conventional therapy in extremely preterm infants: a randomized clinical trial. JAMA Pediatr 2015; 169: 723-730.

14. Oncel MY, Arayici S, Uras N, et al. Nasal continuous positive airway pressure versus nasal intermittent positive pressure ventilation within the minimally invasive surfactant therapy approach in preterm infants: a randomised controlled trial. Arch Dis Child Fetal Neonatal Ed 2016; 101: F323-F328. 